\title{
Fleece-Bound Tissue Sealing in Microvascular Decompression
}

\author{
Levent TANRIKULU1,2, Michael BUCHFELDER ${ }^{2}$, Ramin NARAGHI2,3 \\ ${ }^{1}$ Hannover Nordstadt Hospital, Department of Neurosurgery, Hannover, Germany \\ 'University of Erlangen-Nuremberg, Department of Neurosurgery, Erlangen, Germany \\ ${ }^{3}$ Military Hospital UIm, Department of Neurosurgery, Ulm, Germany
}

\section{ABSTRACT}

AIM: Cerebrospinal fluid (CSF) leakage is a feared complication after microvascular decompression (MVD). In this study, we present our experience of fleece-bound tissue sealing in MVD with an aim to minimize the rate of postoperative CSF leakage.

MATERIAL and METHODS: We treated 50 patients (female/male: 26/24) with neurovascular compression (NVC) syndromes (trigeminal neuralgia, hemifacial spasm and glossopharyngeal neuralgia) by MVD from 2003 to 2006 . All patients underwent retromastoid craniectomy and duraplasty by fleece-bound tissue sealing using the so-called "sandwich technique" by a three-layer reconstruction and cranioplasty.

RESULTS: In 49 (98\%) of 50 patients, we did not observe postoperative CSF leakage. One patient (2\%) suffered postoperative CSF leakage and required surgical revision.

CONCLUSION: Fleece-bound tissue sealing by a three-layer reconstruction is effective and safe in the prevention of cerebrospinal fluid leakage in microvascular decompression.

KEYWORDS: Fleece-bound tissue sealing, Sandwich technique, Retromastoid craniectomy, Microvascular decompression

\section{INTRODUCTION}

$\mathrm{M}$ icrovascular decompression (MVD) for the treatment of neurovascular compression (NVC) syndromes represents an elective intervention in neurosurgery. Neurovascular compression was first observed by Walter Dandy in 1925 during cranial nerve dissections (3).

Peter Jannetta was the first pioneer and introduced the operating microscope in the surgical treatment of trigeminal neuralgia and showed that microvascular decompression is the causal method of surgical treatment (5). As described by Jannetta, there are high requirements for the surgical approach (5). The aspects of surgical closure are especially crucial and can lead to insufficient dural integrity, which can result in the highly feared complication of cerebrospinal fluid (CSF) leakage.
In this study, we present our experience with the technique of fleece-bound tissue sealing in MVD. The goal of this study was to minimize the rate of postoperative CSF leakage.

\section{MATERIAL and METHODS}

\section{Clinical Data}

Fifty patients with NVC syndromes ( 26 female/24 male patients, minimum age 24 years, maximum age 80 years, average age 56.46 years, 41 patients with trigeminal neuralgia, 7 patients with hemifacial spasm and 2 patients with glossopharyngeal neuralgia) were admitted to the Neurosurgical Department of the University Hospital of Erlangen, Germany. All (100\%) underwent MVD. Intraoperative three-dimensional visualization was performed in all 50 cases during the surgical process as has been formerly described (10). 


\section{Operative Technique}

All 50 patients underwent retromastoid craniectomy in the modified supine position with the head turned to the contralateral side after elevation and caudal extension of the ipsilateral shoulder. Craniotomy was performed osteoclastically and cranioplasty was performed by polymethylmetacrylate (PMMA, Palacos $^{\mathrm{TM}}$ ) after dural closure. In all 50 patients, craniotomy was performed by visualizing the junction of the transverse sinus to the sigmoid sinus including the delineation of the transverse and sigmoid sinus margins in order to get a clear and panoramic microsurgical view over the entire cranial nerves from their root exit/entry zones to the exiting foramina at the skull base (Figure 1). There are two ways of performing craniotomy: osteoplastic craniotomy and osteoclastic craniotomy (craniectomy). In our study we performed a craniectomy with delineation of the margins of the transverse to the sigmoid sinus in order to get an extended view over the entire length of the cranial nerves from where they exited the brainstem to the exit foramina at the skull base (Figure 1). Note the different view angles dependent on the craniotomy site: along the trajectory of the red arrow one can look onto the brainstem, along the trajectory of the yellow arrow one can look onto the root entry/exit zones of the cranial nerves and along the trajectory of green arrow one can look into the interfascicular plane between the facial and vestibulocochlear nerves (Figure 1). Intraoperative $3 D$ visualization was interactively performed during surgery in order to compare the microsurgical findings with the rendered high-resolution magnetic resonance imaging data (10) (Figure 2A-F).

Dural closure was performed by the so-called "sandwich" technique in all 50 patients. A three-layer reconstruction was performed. The first inner layer consisted of fleece material (Tachosil $^{\mathrm{TM}}$ ), with the yellow, coated part facing the dura. Whenever possible, the dura was closed primarily and if needed autologous periosteal flaps were used as the second or middle layer of the "sandwich" as a dural replacement. We sutured the dura and the autologous materials by running sutures. The fleece material was then applied as a third, outer layer of the "sandwich" to cover the suture completely and to achieve an absolute watertight closure. We additionally put fleece material on the waxed mastoid air cells. Afterwards we implanted a polymethylmetacrylate flap on the craniectomy field to achieve bony integrity (Figure 3 ). Note that autologous material such as pericranium/galea should be sutured into the leaks within the perforated dura and be strengthened by fleece-bound tissue sealing from the inner dural and outer dural aspect to achieve watertight closure (Figure 4A-D).

\section{RESULTS}

All 50 patients were successfully operated. We did not observe postoperative CSF leakage in 49 of the 50 patients (98\%). The fleece-bound tissue sealing technique was performed in all 50 patients (100\%) without technical problems.

Postoperative CSF leakage was observed on the superior margin of the wound in one patient $(2 \%)$ with trigeminal neuralgia. In this case, there was no evidence of infection with unremarkable lumbar puncture and chemical and microbiological CSF analysis. The CSF leakage was localized below the hearing aid of the patient and the wound was compressed by the patient's hearing aid. Surgical revision was indicated and performed with the application of the fleecebound sealing technique. In 6 of 50 patients (12\%), aseptic nonbacterial meningitis occurred as a foreign body reaction to the Teflon implants.

\section{DISCUSSION}

Microvascular decompression is the causal treatment of choice in patients with neurovascular compression syndromes such as trigeminal neuralgia, hemifacial spasm and glossopharyngeal neuralgia $(1,2,4,5,9)$. High-resolution magnetic resonance imaging and intraoperative application optimizes the anatomical information obtained during surgery (10). Complications such as postoperative CSF leaks, even from clinically undetectable defects, can lead to infection, meningitis, abscess formation, and pneumocephalus (6-8).

Collagen sponges and fibrin glues are popular since they are highly biocompatible and can be used for dural sealing as well as for stopping oozing from tiny vessels (7). A new approach to fast and reliable sealing of dura leakages was introduced with fleece-bound tissue sealing (7). In our series, we used the "sandwich" technique of fleece-bound tissue sealing where we placed the fleece with the active, coated side facing towards the opened dura. The dura was then adapted using running sutures and we used an additional autologous periostal flap for dural replacement. The fleece was then again used to form the outer layer of the "sandwich" before the artificial bone flap of polymethylmetacrylate was implanted.

One of the biggest challenges is watertight dural sealing in neurosurgery. Defects of the posterior cranial fossa often cause CSF fistulas and in cases without detectable leakage, meningitis and abscess formation can aggravate the postoperative clinical course of the patient (7). Autologous grafts

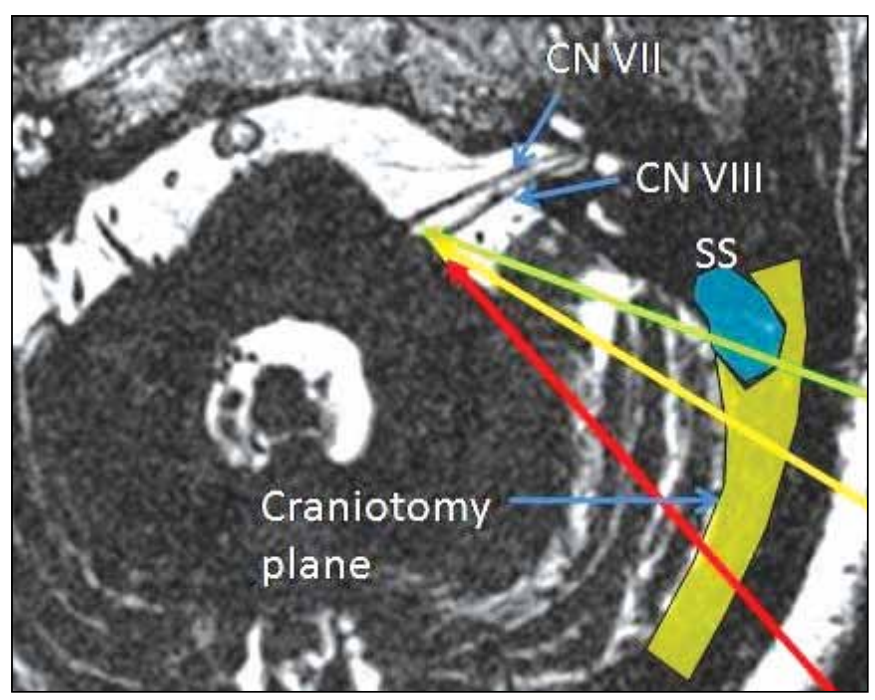

Figure 1: Fleece-bound tissue sealing - Requirements and special points of the approach. (SS: sigmoid sinus, CN VII: facial nerve, CN VIII: vestibulocochlear nerve). 


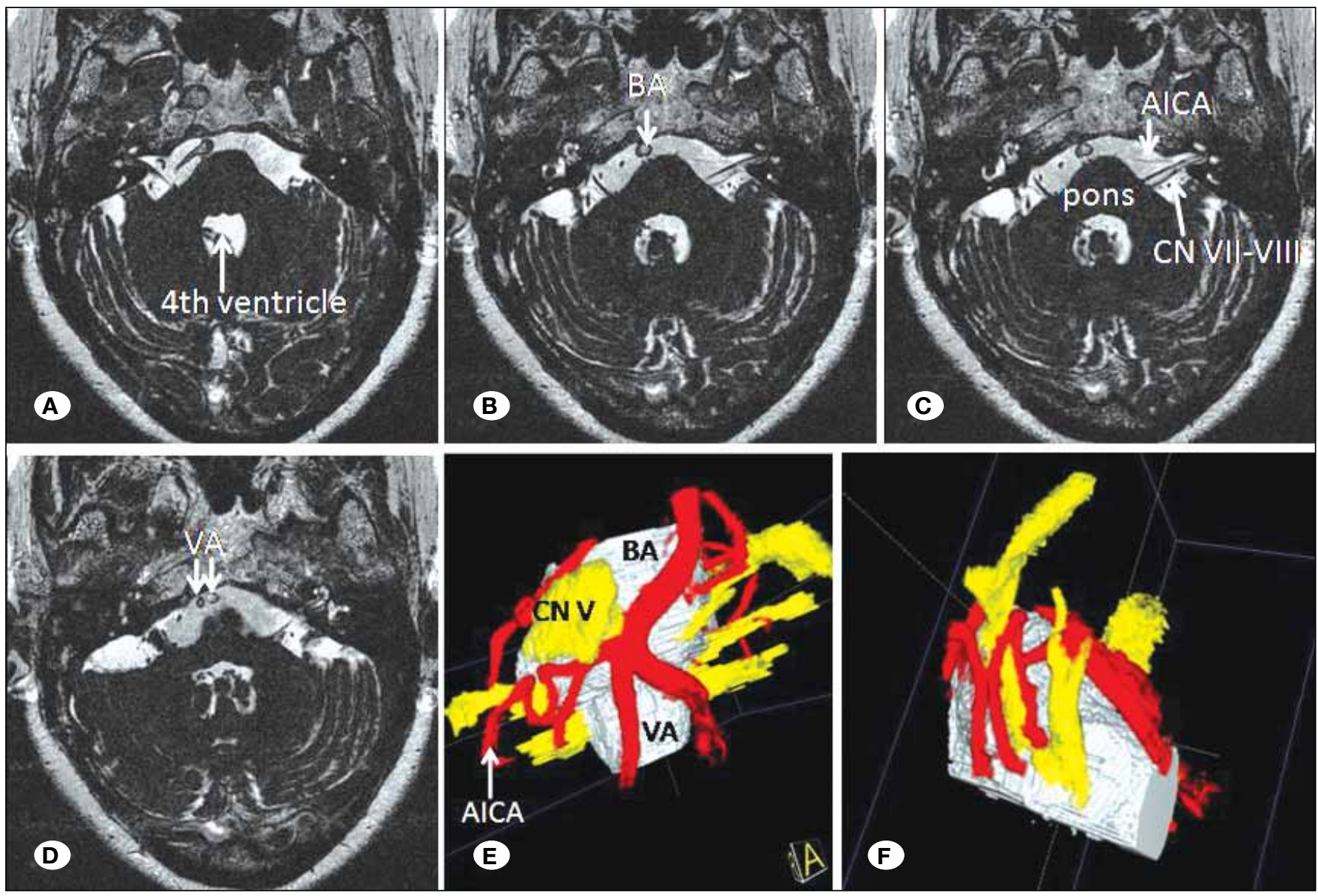

Figure 2: High-resolution T2-weighted magnetic resonance imaging (MRI) with the CISS technique. A-D) High-resolution T2-weighted magnetic resonance imaging with the CISS technique - constructive interference in the steady state- with high contrast between cranial nerves and corresponding tiny vasculature. E-F) Three-dimensional visualization of the MRI-CISS data by direct volume rendering with panoramic view over the essential and individual neurovascular relationships in a patient with right-sided Hemifacial Spasm and neurovascular compression of the root entry/exit zone of the right-sided facial nerve by a vascular loop of the AICA. (AICA: Anterior inferior cerebellar artery, BA: basilar artery, VA: vertebral artery, CN V: trigeminal nerve, CN VII: facial nerve, CN VIII: vestibulocochlear nerve).

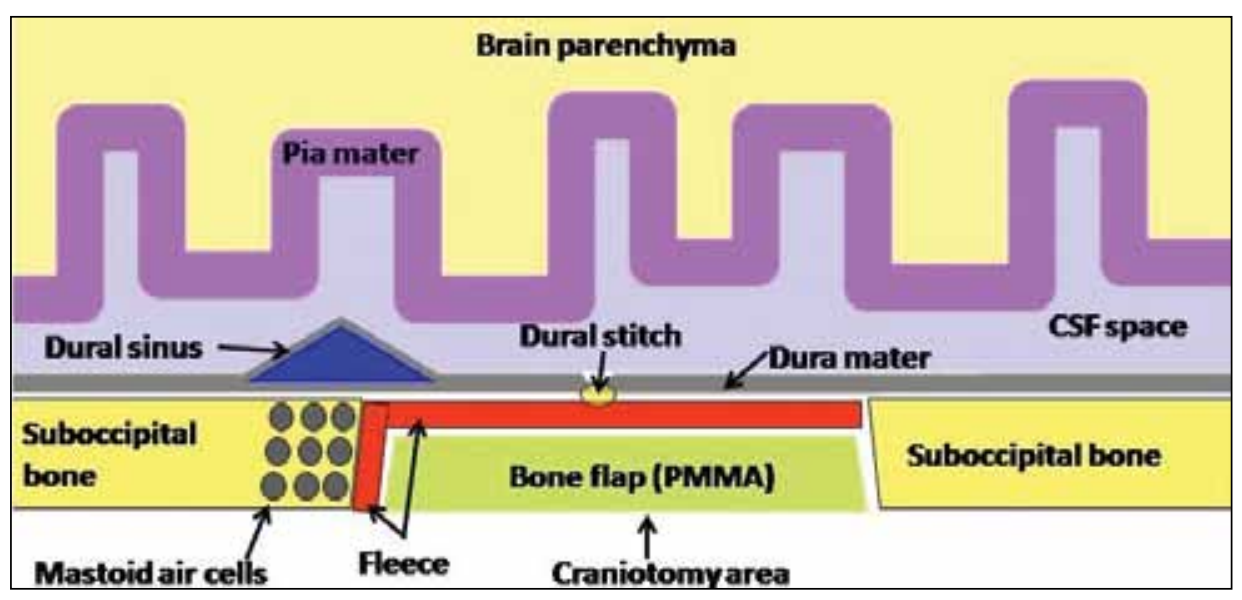

Figure 3: Fleece-bound tissue sealing technique with the "sandwich" model. 


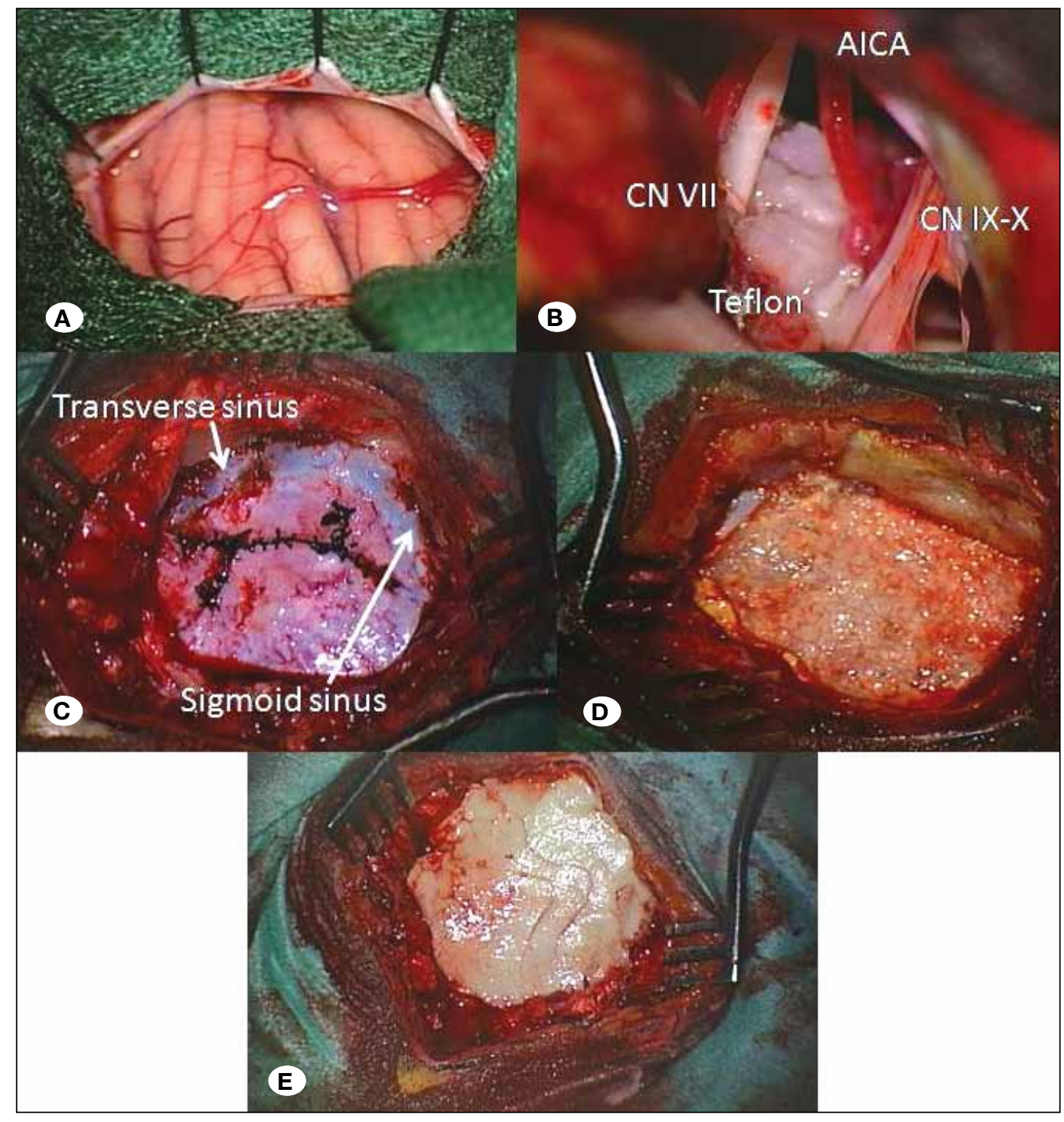

Figure 4: Microvascular decompression with Teflon after mobilization and decompression of a vascular loop of the anterior inferior cerebellar artery.

A) Microsurgical view onto the cerebellar surface after suboccipital, retromastoid, retrosigmoidal craniectomy for right-sided Hemifacial Spasm. B) Microvascular decompression with Teflon after mobilization and decompression of a vascular loop of the anterior inferior cerebellar artery (AICA)(CN VII: facial nerve, CN IX: glossopharyngeal nerve, $\mathrm{CN} \mathrm{X}$ : vagus nerve. C) Note the envelopeshaped dural opening/ suture after the application of fleece-bound material on the inner side of the dura, dural sutures and then on picture. D) fleece-bound sealing material on the outer side of the dura and the waxed mastoid air cells. E) polymethylmetacrylate implant. such as fascia lata, temporalis fascia or pericranium have proved to be suitable as a dural substitute but they are not always available. Liquid sealants such as fibrin glue require a dry and horizontal application surface to polymerize which is not always possible in areas of dural leakage (7). With the technique of fleece-bound tissue sealing, we think that this method is an adequate alternative option for dural substitution and has hemostatic effects. Fleece-bound sealing is easy to handle, is available quickly and has high clinical acceptance. It is safe, watertight, and efficient. The main advantage is its surgical usage without suturing. No infections occurred when we used the material. The observed nonbacterial infections were due to foreign body reaction to the Teflon implants more than to the used fleece material. The main disadvantage of the fleece-bound material is the high cost.

We personally do not think that the use of fleece material only can ensure dural watertightness, because we think that first of all a watertight closure in the posterior cranial fossa must be performed by autologous grafts such as pericranium and galea and has to be sealed with fleece materials from inside and outside the dura to secure the pressure of CSF against the autologous dural material. In our experience, we performed an envelope-shaped dural opening so that the dural edges would not markedly shrink during long surgeries in contrast to semilunar openings of the dura. It took 10 years to submit the data about our technique of fleece-bound surgery, because our clinical-scientific research primarily focused on the imaging and image processing of neurovascular compression syndromes of the treated syndromes such as trigeminal neuralgia, hemifacial spasm and glossopharyngeal neuralgia for the last few years.

\section{- CONCLUSION}

The fleece-bound tissue sealing technique is an adequate technique for dural reconstruction in posterior fossa surgery. It has excellent tissue-sealing properties and is capable of stopping even heavy bleeding from dural sinuses. 


\section{REFERENCES}

1. Amagasaki K, Watanabe S, Naemura K, Shono N, Nakaguchi $\mathrm{H}$ : Safety of microvascular decompression for elderly patients with trigeminal neuralgia. Clin Neurol Neurosurg 141:77-81, 2016

2. Burchiel KJ: A new classification of facial pain. Neurosurgery 53: 1164-1167, 2003

3. Dandy WE: Trigeminal neuralgia and trigeminal tic douloureux. In: Lewis D (ed). Practice of Surgery. Hagerstown, MD: WF Prior CO, 1932: 177-200

4. Hitchon PW, Zanaty M, Moritani T, Uc E, Pieper CL, He W, Noeller J: Microvascular decompression and MRI findings in trigeminal neuralgia and hemifacial spasm. A single center experience. Clin Neurol Neurosurg 139:216-220, 2015

5. Jannetta PJ: Arterial compression of the trigeminal nerve at the pons in patients with trigeminal neuralgia. J Neurosurg 26: 159-162, 1967
6. Lee MH, Jee TK, Lee JA, Park K: Postoperative complications of microvascular decompression for hemifacial spasm: Lessons from experience of 2040 cases. Neurosurg Rev 39(1):151-158, 2016

7. Matula CW, Steiger NS: Hemostasis and fleece-bound sealing in neurosurgery. Stuttgart: Georg Thieme Verlag, 2005

8. Montano N, Conforti G, Di Bonaventura R, Meglio M, Fernandez E, Papacci F: Advances in diagnosis and treatment of trigeminal neuralgia. Ther Clin Risk Manag 11:289-299, 2015

9. Sindou M, Howeidy T, Acevedo G: Anatomical observations during microvascular decompression for idiopathic trigeminal neuralgia (with correlations between topography of pain and site of the neurovascular conflict). Prospective study in a series of 579 patients. Acta Neurochi (Wien) 144: 1-12, 2002

10. Tanrikulu L, Hastreiter P, Troescher-Weber R, Buchfelder M, Naraghi R: Intraoperative three-dimensional visualization in microvascular decompression. J Neurosurg 107(6):11371143,2007 